\title{
Formation of the universe: From classical to quantum cosmology
}

\section{H. Nicolai and M. Pössel}

Albert-Einstein-Institute/MPI for Gravitational Physics, Am Mühlenberg 1, 14476 Golm Germany

nicolai@aei.mpg.de

Received 25 Dec. 2000, accepted 31 Dec. 2000 by C. Thomsen

Abstract. We give a brief overview of the role of quantum theory in cosmology, reviewing the standard model of classical cosmology, inflation and quantum cosmology.

Keywords: cosmology, inflationary models, quantum cosmology

PACS: $98.80,04.60,98.80 . \mathrm{H}$

\section{Introduction}

Quantum theory makes numerous important contributions to cosmology, the study of the universe as a whole: Within the standard model of cosmology based on Einstein's theory of general relativity, the quantum field theories of electroweak and strong interactions are used to describe the properties of matter in the high-temperature phases of the universe. In addition, it has been theorized that prior to the evolution predicted by the standard model of cosmology, our universe has undergone an inflationary period of rapid exponential expansion; the models for this inflation also rely on quantum theoretical arguments. Finally, one can attempt to view the universe as a whole as a quantum system; this is commonly referred to as quantum cosmology. A review as brief as this cannot attempt to present even a moderately complete bibliography of original papers; rather, the references that we give should be viewed as suggestions for further reading. A few more general references for the topics treated here are Refs. [1].

\section{The cosmological standard model}

There is observational evidence that viewed on a scale of several hundred million lightyears, our universe is homogeneous and isotropic, and furthermore, that it is in a process of uniform expansion. Newtonian gravity cannot account for this. Instead, modern cosmology [2] is based on Einstein's description of gravity as governed by one of the most elegant systems of equations in physics, the Einstein field equations:

$$
R_{\mu \nu}-\frac{1}{2} g_{\mu \nu} R=8 \pi G T_{\mu \nu}-\Lambda g_{\mu \nu} .
$$


These equations relate the geometry of spacetime (described by the metric $g_{\mu \nu}$, the Ricci tensor $R_{\mu \nu}$ and the Ricci scalar $R$ on the left-hand side) to the energy and momentum of the matter contained within spacetime (in the form of the energymomentum tensor $T_{\mu \nu}$ on the right-hand side); $G$ is Newton's constant. The cosmological constant $\Lambda$ is an addition to the original field equations that can be interpreted as an energy density associated with empty space itself. Spacetime is no longer a stage on which the drama of matter interactions unfolds; instead, it takes an active part in the dynamics.

As non-linear partial differential equations, the Einstein equations are extremely difficult to solve. However, under the assumption of homogeneity and isotropy of the universe, one can choose a natural coordinate system at rest with respect to the uniformly distributed matter ("co-moving coordinates") from which follows a metric (which characterizes the space-time geometry by relating infinitesimal coordinate differences to an infinitesimal distance $d s$ ), the Robertson-Walker metric

$$
d s^{2}=-d t^{2}+a^{2}(t)\left(\frac{d r^{2}}{1-k r^{2}}+r^{2} \mathrm{~d} \Omega^{2}\right) .
$$

This contains only one unknown function, the cosmic scale factor $a(t)$ which depends solely on the time coordinate (the "cosmic time") and shows how the spatial distance between two objects changes with the cosmic expansion: if their initial distance at cosmic time $t_{0}$ was $d\left(t_{0}\right)$, then their distance at a later time $t_{1}$ will be $d\left(t_{1}\right)=a\left(t_{1}\right) / a\left(t_{0}\right) \cdot d\left(t_{0}\right)$. The constant $k$ can take on one of three possible values, corresponding to different types of cosmological models. For $k=1$, the spatial part of the universe is locally spherical, analogously to the surface of a sphere, for $k=0$ it is locally flat analogously to a plane, and for $k=-1$ it is locally hyperbolic, analogously to a saddle. The scale factor can be related to observations; in particular, by measuring the red-shifts and distances of distant galaxies, one can determine the current expansion rate $\dot{a} / a$, which is the so-called Hubble constant $H_{0}$, as well as the current value of $-\ddot{a} / \dot{a}^{2}$, known as the deceleration parameter $q_{0}$. Recent numerical values for these parameters are in the range of $H_{0}=60$ to $80 \mathrm{~km} /(\mathrm{s} \mathrm{MPc})$ and $q_{0}<0$, pointing to an accelerated expansion.

The matter in such a universe has the energy momentum tensor of an ideal fluid, $T_{\mu \nu}=\operatorname{diag}(\rho, p, p, p)$, with characteristic properties encoded into an equation of state $p=p(\rho)$. Relevant for different eras of our universe are (non-relativistic) matter with the equation of state $p=0$ and radiation with $p=1 / 3 \rho$. For pure vacuum energy, $p=-\rho$. Substituting the energy-momentum-tensor and the metric Ansatz (2) into Einstein's field equations, one obtains (either when $\Lambda=0$ or including the energy contribution of $\Lambda$ in $\rho$ and $p$ )

$$
\frac{\ddot{a}}{a}=-\frac{4 \pi G}{3}(\rho+3 p) \quad \text { and } \quad\left(\frac{\dot{a}}{a}\right)^{2}+\frac{k}{a^{2}}=\frac{8 \pi G}{3} \rho .
$$

A spacetime with the metric Eq. (2), satisfying these equations, is called FriedmannLemaitre-Robertson-Walker (FLRW) universe. For the sample equations of state shown above, we can solve Eq. (3) and write down the relation between the scale factor and the matter density, as well as the scale factor's dependence on cosmic time: 


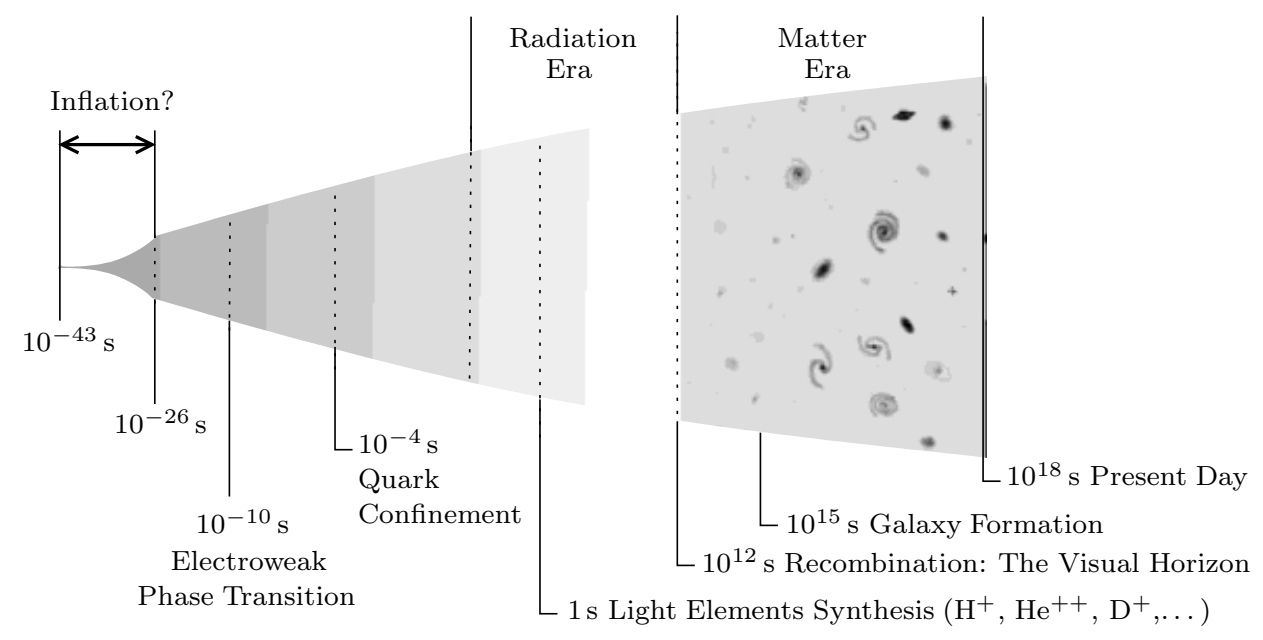

Fig. 1 Development of the universe from the big bang to the present (schematic)

For a matter-dominated universe, $\rho \propto a^{-3}$ and $a(t) \sim t^{2 / 3}$; for a universe dominated by radiation, $\rho \propto a^{-4}$ and $a(t) \sim t^{1 / 2}$. It turns out that the total mass density $\rho$ of the universe directly determines its local geometrical properties. Define the critical density as $\rho_{\text {crit }}:=3 H_{0}^{2} / 8 \pi G$, and define $\Omega:=\rho / \rho_{\text {crit }}$. Then for $\Omega>1$, the universe is locally spherical; for $\Omega=1$ it is locally flat; and for $\Omega<1$, it is locally hyperbolic. For the values of the Hubble constant $H_{0}$ given above, the critical density is $\rho_{\text {crit }} \sim 10^{-29} \mathrm{~g} / \mathrm{cm}^{3}$. The observations point to $\Omega$ remarkably close to 1 . However, drawing together mass estimates from observations of matter dynamics (for instance, the rotation curves of galaxies) and from primordial nucleosynthesis (see below), it appears as if substantial contributions to $\Omega$ are made by so-called non-baryonic dark matter, a hypothetical, as yet unknown form of non-standard matter that does not take part in electromagnetic interactions. Typical estimates are $\Omega_{b} \approx 0.05$ for ordinary, baryonic matter, $\Omega_{n b} \approx 0.3$ for baryonic and non-baryonic matter combined, and $\Omega_{\Lambda} \approx 0.7$ for the energy density associated with the cosmological constant.

Ordinary matter obeys $\rho+3 p>0$ which, by Eq. (3), implies permanent deceleration $\ddot{a}<0$ and leads to solutions for which, some finite time $t_{0}$ ago, $a=0$. This extreme point in the development of FLRW models is commonly called the big bang. The standard model of cosmology describes the universe's evolution from about $10^{-26} \mathrm{~s}$ after the big bang to the present (Fig. 1). An important - and successful - prediction of standard cosmology is that of the existence of the cosmic background radiation (CBR), blackbody radiation made up of photons that have not interacted with the rest of the universe since the time of recombination near $t \approx 10^{12} \mathrm{~s}$. The isotropy of the CBR is further evidence of the homogeneity and isotropy of our universe, and a very active area of research is that of structure formation [4], addressing the question of how the observed inhomogeneous structures of our universe (e.g., galaxy clusters) developed from such nearly homogeneous initial conditions. 
Within the cosmological standard model, quantum theory, or more precisely, the standard model of elementary particles, plays a crucial role $[1,3]$. The standard model of particle physics contributes to the predictions for primordial nucleosynthesis at cosmic time $t \approx 1 \mathrm{~s}$; the fact that these predictions of the relative abundance ratios for light nuclei such as Helium-4, Deuterium and Lithium-7 agree with observation is one cornerstone of supporting evidence for the standard cosmology. Another important question is that of baryogenesis: why does the universe around us consist predominantly of matter, instead of an even mix of matter and anti-matter? While the issue is not completely resolved, there exist a number of viable models for how this matterantimatter-asymmetry developed [3].

For all its predictive power, the standard model of cosmology leaves open a number of important questions. First of all, the singularity problem: What happened at $t=0$ (or "before")? The answer presumably lies with a quantum theory of gravity (Sect. 4). Secondly, elementary particle physics would appear to predict a very large cosmological constant. But then, why is the observed value for the constant so comparatively small? Furthermore, the uniqueness problem: Is there a reason for the observed properties of our particular universe? This is especially pressing in the light of superstring theory, which seems to imply a huge number $\left(10^{10^{*}}\right)$ of possible consistent vacua. Two particular properties are especially interesting: Why is $\Omega$ so close to one, corresponding to a flat universe (flatness problem) or, put in a different way, why is the extension of the universe so large, compared with the Planck length, the natural length scale $\ell_{\text {Planck }}=\sqrt{G \hbar / c^{3}}$, why is $R_{\text {univ }} \sim 10^{28} \mathrm{~cm} \gg \ell_{\text {Planck }} \sim 10^{-33} \mathrm{~cm}$ ? Why is our universe isotropic and homogeneous in the first place? This last question is made especially vexing by the so-called horizon problem: in the standard cosmological models, regions of the cosmic background radiation that we observe as homogeneous should not even have been in causal contact with each other - in the early universe, there should have been a myriad of causally disconnected regions.

\section{Inflation}

Inflationary models postulate that the evolution of our universe as described by FLRW models was preceeded by an inflationary phase of rapid exponential expansion [5]. This assumption is motivated by the problems of flatness and homogeneity, by the hope that quantum fluctuations might provide the seed for later structure formation, and by the attempt to explain the observed scarcity of certain exotic relic particles predicted by some models for the very early, high energy cosmos. Such an expansion is characteristic for a universe dominated by vacuum energy embodied in a cosmological constant $\Lambda>0$. Defining $\Lambda=8 \pi G \lambda$, one can view the cosmological constant as the presence of "matter" with the equations of state $\rho=\lambda=$ const. and $p=-\rho=-\lambda$. As opposed to ordinary matter, we have $\rho+3 p<0$ which, by Eq. (3), entails $\ddot{a}>0$. For times $t \gg 0$ the evolution of the cosmic scale factor approaches $a(t) \sim e^{H_{0} t}$ with $H_{0}=\sqrt{\Lambda / 3}$.

Inflation can be achieved in an elegant way with the help of quantum field theory, namely as a consequence of the presence of a scalar field known as the inflaton in the early universe. The generic Lagrangian of a scalar field is $\mathcal{L}=1 / 2 g^{\mu \nu} \partial_{\mu} \varphi \partial_{\nu} \varphi-V(\varphi)$, 


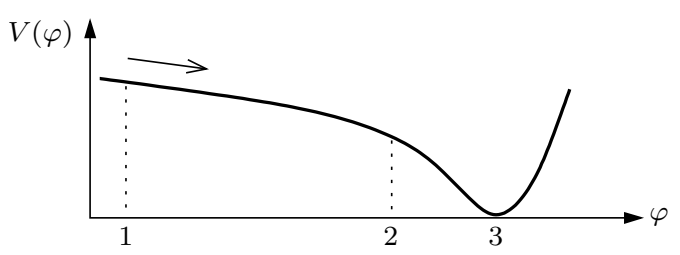

Fig. 2 Generic potential for the inflaton field

corresponding to an energy-momentum tensor $T_{\mu \nu}=\partial_{\mu} \varphi \partial_{\nu} \varphi-g_{\mu \nu} \mathcal{L}$. From this it can be read off that for a near-constant scalar field, $\rho=-p=V(\varphi)$. Apparently, such a field can provide the matter behaviour needed for the inflationary phase. For simplicity, we assume that the scalar field evolves in an FLRW background, and that $\varphi(t, \boldsymbol{x}) \equiv \varphi(t)$. Then its equation of motion has the simple form

$$
\ddot{\varphi}+\frac{\mathrm{d} V}{\mathrm{~d} \varphi}+3 \frac{\dot{a}}{a} \dot{\varphi}+\Gamma \dot{\varphi}=0,
$$

where the first three terms follow from the given Lagrangian while the last term incorporates the interaction with other matter fields, which will become important later on. The mechanical analogue would be a particle moving in the potential $V$ with friction proportional to its velocity. Viewing the field classically (i.e., considering its expectation value $\langle\varphi\rangle$ ), we can use this equation to describe the field dynamics in a typical inflation scenario. A generic potential for the inflaton field is shown in Fig. 2; important is the presence of a minimum at 3 and of a comparatively flat stretch between 1 and 2 . The initial inflaton expectation value is taken to be at point 1 . Then, $\langle\varphi\rangle$ slowly evolves towards point 2; during this "slow rolling" phase, the scalar field is approximately constant and drives inflation in the way indicated above. A typical value for the duration of this phase is $\Delta t \sim 10^{-26} \mathrm{~s}$ (a long time, compared to the expansion time scale $H_{0}^{-1}$ ), during which $a$ increases by a factor of about $10^{30}$. When, continuing down the slope, the expectation value has reached the minimum at 3 , the scalar field begins to oscillate. This is the so-called reheating phase in which the term $\Gamma \dot{\varphi}$ becomes important. As mentioned above, it contains the coupling of $\varphi$ to all other matter degrees of freedom, and in the reheating phase, the scalar field expends its energy in pair creation of matter particles (or the decay of $\varphi$-particles into ordinary matter), leading to a high-temperature universe which then continues its evolution as described by the FLRW-models. In a natural way, the inflationary phase leads to a flat, homogeneous universe: Possible initial inhomogeneities get "inflated away", the inflation naturally leads to $\Omega$ of order unity, and the different parts of the CBR we observe today have been in causal contact at sufficiently early times.

At present, inflation is not "a" model, but rather a framework into which a plethora of models fit - models differing, for instance, by the choice of potential $V(\varphi)$, but all reproducing the basic stages of slow rolling and reheating. A class of models that enjoys great current popularity embodies what is called chaotic inflation. These models assume a primordial cosmos full of violent quantum fluctuations, in some regions of which the expectation value of the scalar field has a value that permits inflationary 
expansion. Such a region will inflate, forming a huge bubble. At least for some time, the inner parts of this bubble will be causally unconnected to the rest of the universe, and it is inside such a bubble that the whole of our observable universe is thought to be situated. The uniqueness problem has been circumvented - at the cost that, now, our observable universe is just one of a myriad of other "universes", a tiny part of a cosmos possibly $10^{10^{100}}$ times larger.

While inflation appears to be an elegant way of overcoming the flatness and homogeneity problems, it introduces some new problems of its own. The multitude of models shows us that our knowledge of a possible inflationary phase is still rather vague. Generally, the inflaton field is introduced $a d-h o c$, and does not arise in a natural way from some basic elementary particle model. Still worse, the fundamental theories one could expect to govern the quantum gravity phase directly preceeding inflation appear to prefer a negative cosmological constant, $\Lambda<0$ (e.g., Anti-deSitter space). While there appear to be a few "stable" predictions shared by all inflationary models and consistent with available data (in addition to the flatness of the universe, a certain spectrum of density fluctuations in the cosmic background radiation; additional predictions of relic gravitational waves might become testable in the near future), inflation also introduces a great number of as-yet untestable predictions (and, in the case of multiple "universe bubbles", a plethora of spacetime regions of our universe that cannot, even in principle, be observed today or in the foreseeable future as they concern space-time regions behind the current event horizon). Ultimately, one must still strive to understand what "happens" at the Planck scale. Given that, typically, the initial value of the inflaton field falls into that energy region, an understanding of Planck-scale physics is now important for setting the initial conditions of inflation and showing how the preconditions of inflation - a universe dominated by the inflaton field - can arise in the first place.

\section{Quantum cosmology}

Again and again, the preceeding sections have led us to the question of what a quantum theory of gravity looks like, and how it might influence the development of the very early universe. As yet, no conclusive answer to that question is known. In the search for such a theory, there are currently two major approaches: Starting from elementary particle physics, there is superstring theory [6], which might lead not only to a theory of quantum gravity, but to a unified theory of all interactions. The most immediate consequence of this theory is the presence of additional particle modes in the very early universe, while recent developments concerning possibly large extra dimensions and hints of an underlying non-commutativity of space-time could well have a more fundamental cosmological significance. Here, however we will focus on another Ansatz, the program of canonical quantization of gravity [7], which attempts to apply a quantization procedure to Einstein's theory of gravity that respects the theory's geometric nature. Within this latter framework, quantum cosmology [8] is an attempt to explain the evolution of the universe as a whole in quantum mechanical terms.

One basic conflict between general relativity and ordinary quantum mechanics is already apparent in the quantum mechanics of point particles, where the dynamics 
of a state vector $\Psi\left(t, q_{1}, \ldots, q_{N}\right)$ is described by the Schrödinger equation and where $\left|\Psi\left(t, q_{1}, \ldots, q_{N}\right)\right|^{2}$ has a straightforward interpretation as a probability density. In the Schrödinger equation, time plays a special role and is not quantized, in contrast to the space coordinates which are replaced by operators obeying certain commutation relations. This special split in space and time is incompatible with the general coordinate invariance that relativity demands, and for relativistic point-particle mechanics, a different formulation is needed. The solution is to start from an invariant pointparticle action defined by the invariant length of the particle's world line, namely the action $S=m \int d s=m \int \sqrt{\dot{x}^{\mu} \dot{x}_{\mu}} \mathrm{d} \tau$, with $\tau$ some variable parametrizing the world line. Define a conjugate momentum $p_{\mu}:=\partial \mathcal{L} / \partial \dot{x}^{\mu}$. The invariance of the action under reparametrizations $\tau \rightarrow \tau^{\prime}=\tau^{\prime}(\tau)$ leads to the constraint $\mathcal{H}:=p_{\mu} p^{\mu}-m^{2}=0$. If we quantize the system, $p_{\mu} \mapsto-i \partial_{\mu} \equiv-i \partial / \partial x^{\mu}$, the quantum version of this constraint will have to be imposed on all states, $\hat{\mathcal{H}} \phi=-\left(\partial^{\mu} \partial_{\mu}+m^{2}\right) \phi=0$. This (Hamiltonian) constraint is in fact the Klein-Gordon equation which determines the field's dynamics. We have achieved parametrization invariance, but at a cost: it is no longer possible to interpret $\phi^{*} \phi$ as a probability density because it is not conserved. The charge constructed from the current $j^{\mu}=i\left[\phi^{*} \cdot \partial^{\mu} \phi-\partial^{\mu} \phi^{*} \cdot \phi\right]$ is conserved, but not positive and thus not a good probability density either. The resolution demands a radical re-interpretation and results in a quantum field theory in which the $\phi(x)$ become operators themselves.

The situation for quantizing a whole spacetime, structured by its metric field $g_{\mu \nu}$, is somewhat similar. As a first step, one foliates spacetime into slices of "equal time", corresponding to a split of spacetime into space and time. Continuing the canonical analysis for the configuration space variables, which are the space-space components $g_{i j}$ of the metric, and their conjugate momenta, one finds that Einstein's equations lead to four constraint equations - three that arise from the reparametrization freedom for each space slice and one that arises from the possibility of small deformations orthogonal to the slice. The latter gives rise to the Wheeler DeWitt (WDW) equation

$$
\left(G_{i j, k l}(\boldsymbol{x}) \frac{\delta}{\delta g_{i j}(\boldsymbol{x})} \frac{\delta}{\delta g_{k l}(\boldsymbol{x})}+\sqrt{g(\boldsymbol{x})} R^{(3)}(\boldsymbol{x})\right) \Psi\left\{g_{i j}(\boldsymbol{x})\right\}=0,
$$

where $G_{i j, k l}$ is a metric on the space of all 3-metrics, and $\Psi$ is called the "wave function of the universe". The fact that this is a Hamiltonian constraint rather than a Hamiltonian reflects the general covariance of the theory. The situation is, however, much more complicated than in the case of the point particle: as it stands, Eq. (5) is ill-defined; the choice of Hilbert space is much less obvious, and it is not clear whether or not Eq. (5) will become a well-defined operator valued distribution. In general, the WDW equation leads to the problem of time: To start with, it gives us a completely static description of the universe; the wave function $\Psi$ contains all the information "from beginning to end". Time can only be introduced intrinsically: the WDW equation is hyperbolic (in field space); thus, one of the configuration space variables could serve as a time coordinate (a "clock field"), leading to the emergence of time from a "timeless" equation. Again, this is similar to the situation of the relativistic point particle, where the world-line parameter is absent from the dynamical constraint equation. A more general problem in quantum cosmology is that the usual Copenhagen interpretation is not applicable anymore. The observer is now an integral 
part of the quantum system. Still unsolved is the problem of decoherence - how does a classical (FLRW or inflationary) cosmology emerge from $\Psi$ ? This question has in fact prompted new interpretations of quantum mechanics, such as the consistent history formulation of [9].

While many questions of interpretation remain unanswered, the last decades have seen both improvements in the mathematical formulation of canonical quantum gravity (Ashtekar's new variables, loop formulation) and progress toward a better understanding of quantum cosmology in this framework.

\section{Outlook}

Many fundamental questions about the role of quantum theory in cosmology remain unsolved. While progress has been made and is being made, we are still far from understanding the quantum basis of our universe. We can but hope to reach eventually such an understanding - aided by theorists providing us with a proper framework for quantum gravity, and by experimentalists and observers collecting new data, e.g., about gravitational waves, dark matter and new (supersymmetric?) particles.

\section{Acknowledgement}

We thank W. Buchmüller, J. Ehlers, T. Thiemann and A. Butscher for helpful comments and suggestions.

\section{References}

[1] G. Börner, The Early Universe, Springer, Berlin 1988; E. W. Kolb and M. S. Turner, The Early Universe, Addison-Wesley, Reading, Mass. 1990; J. A. Peacock, Cosmological Physics, Cambridge Univ. Press, Cambridge 1999; P. J. E. Peebles, Principles of Physical Cosmology, Princeton Univ. Press, Princeton 1993

[2] S. Weinberg, Gravitation and Cosmology, Wiley, New York 1972; C. W. Misner, K. S. Thorne and J. A. Wheeler, Gravitation, Freeman, San Francisco 1973

[3] W. Buchmüller, this volume; W. Buchmüller and M. Plümacher, hep-ph/0007176; A. Riotto and M. Trodden, Ann. Rev. Nucl. Part. Sci. 49 (1999) 35 [hep-ph/9901362]

[4] T. Padmanabhan, Structure formation in the universe, Cambridge Univ. Press, Cambridge, 1995

[5] A. D. Linde, Particle Physics and Inflationary Cosmology, Harwood Academic, Chur 1990; K. A. Olive, Phys. Rep. 190 (1990) 307

[6] J. G. Polchinski, String theory (2 vols.), Cambridge Univ. Press, Cambridge 1999,2000; M. B. Green, J. H. Schwarz and E. Witten, Superstring theory (2 vols.), Cambridge Univ. Press, Cambridge 1987

[7] A. Ashtekar, "Non-Perturbative Canonical Gravity", Lecture notes prepared in collaboration with R.S. Tate, World Scientific, Singapore 1991

[8] D.L. Wiltshire in Cosmology: the physics of the universe edited by B. A. Robson, World Scientific, Singapore 1996, p. 473; C. Kiefer in Towards Quantum Gravity edited by J. Kowalski-Glikman, Springer, Berlin 2000, p. 158 [gr-qc/9906100]

[9] J. B. Hartle Gravitation and Quantizations edited by B. Julia and J. Zinn-Justin, Elsevier, Amsterdam 1995, p. 285 